\title{
Research Ethics and Participatory Research in an Interdisciplinary Technology-Enhanced Learning Project
}

\begin{abstract}
This paper identifies some of the tensions that became apparent in a large interdisciplinary technology enabled learning project as its members attempted to maintain their commitment to responsive, participatory research and development in naturalistic research settings while also 'enacting' these commitments in formal research review processes. It discusses how these review processes were accompanied by a commitment to continuing discussion and elaboration across an extended research team and to a view of ethical practice as an aspect of phronesis or 'practical wisdom' which demands understanding of specific situations and reference to prior experience. In this respect the interdisciplinary nature of the project allows the diverse experience of the project team to be brought into play, with ethical issues a joint point of focus for continuing interdisciplinary discourse.
\end{abstract}

Keywords: Ethics: interdisciplinary; participatory research; phronesis; community of inquiry

\section{Introduction}

This paper discusses some of the challenges faced by a major interdisciplinary technology-enhanced learning project as it has developed an ethical framework that will inform its research activities and patterns of engagement with a range of participants in different settings and with different disciplinary practices. "Ensemble: Semantic Technologies for the Enhancement of Case Based Learning" project runs for three years from October 2008 to 2011 as one of eight major projects funded under the joint ESRC and EPSRC Technology Enhanced Learning Phase of the broader Teaching and Learning Research Programme (Pollard 2007). The project is exploring the potential of technologies associated with the emerging 'semantic web' (Berners-Lee et al. 2001; Shadbolt et al.2006) to support teaching in complex, controversial and rapidly-evolving fields where case based learning is the pedagogical approach of choice. The semantic web is envisaged as a linked 'web of data' in which heterogeneous resources (qualitative and quantitative, images, audio, video and text) are made available so that users (in this case, teachers and students in higher 
education) can engage with them in creative and generative ways. As such it represents a major programme of technological development that brings with it a range of new pedagogical opportunities and challenges.

The project's research activities involve the participation of teachers and students in undergraduate and postgraduate courses in two distinctive higher education institutions. It seeks to explore both the nature and role of the cases around which learning is focused, and the part that emerging technologies and techniques can play in supporting this learning. The project has a number of features, which presented particular challenges for research design, methodologies and ethical practice across the project.

Among these challenges are the following:

- Diversity in settings and research perspectives: while all of the project research settings use some kinds of 'cases' in teaching and learning, these have different roles, structure and scope, and the pedagogical practices and discourses that accompany them are widely varied (Tscholl, Tracy and Carmichael 2009). At the same time, the research team themselves have brought with them differing experiences and commitments, enacted through varied research practices (Rimpilainen and Edwards (2009) provide a detailed account of how different 'hinterlands' were enacted in a single research setting).

- The use of technology in the research process: from the outset the project has used a range of technologies and techniques in order to collect data and to support interpretation and analysis. These 'technology enhanced research' approaches have offered the potential for researchers to gather new forms of data (such as logs of online activity); to amass large volumes of data collected over long periods; and 
to carry out primary and secondary analysis in novel ways to which provision of fully 'informed consent' might not be possible.

- Commitments to participatory design and participatory research: from the outset there has been a commitment on the part of the project to involve participants not just as respondents or in the generation of technical 'user requirements', but also in recruitment of other participants; research design; design and evaluation of technological tools; co-interpretation and sense-making of data; and in the dissemination and sustainability of the work of the project. This has not only offered greater opportunities to participants to inform and shape the project, but has also placed upon them a new range of responsibilities, particularly when involved in innovations that could potentially change the experience of other teachers or learners.

- Directed but unpredictable changes in pedagogical practice and technologies: following from the participatory nature of the project, it was understood that while not conceived as a programme of technological 'interventions' within a quasiexperimental framework, part of the work of the project would be the development of new technological tools which would be introduced into teaching and learning environments. These would clearly change practices, discourses and relationships in those environments and had the potential to have impacts on learning outcomes. The engagement of participants with the work of the project over an extended period also had the potential to frame their reflections on existing practice, inform innovations, and change relationships within and beyond formal classroom settings.

We shall return to these themes in relation to processes of ethical review and research practices in different settings later in this paper. 


\section{The Project as an Interdisciplinary Community of Inquiry}

A particular challenge and strength of the Ensemble research group is that the diversity of the projects' research settings is matched by the diversity of the project team, which includes researchers with backgrounds in computer sciences, information science, cognitive sciences, social sciences and education. It also includes disciplinary specialists with interests in teaching and learning in specific disciplinary settings; two linked $\mathrm{PhD}$ students conducting their own projects; and student researchers who work with the project under an Undergraduate Research Opportunities Scheme (UROP). Many of the research team have previously worked in multiple disciplinary or professional settings (for example, former scientists now working as educational researchers; social scientists who currently work on information science projects; and teachers who are now learning technologists). While some characterise themselves as 'discipline-hoppers' or 'boundary-crossers' (echoing Wenger's (1998) notion of 'brokers' as belonging to multiple communities of practice and being able to participate in the meaning-making practices of each) what is evident is a variety of what Hall (1999) calls 'personal pan-disciplinarity' which is common in emerging fields such as technology-enhanced learning.

Working across disciplines has often been understood as involving the bringing together of teams of individuals with diverse expertise in order to solve comparatively well-defined (or at least predefined) problems; so the designer or manager of an educational software development project might identify the need for educational researchers, systems analysts, programmers, user interface designers and so on. A distinction is sometimes drawn between this kind of multidisciplinary activity and interdisciplinary research (Austin, Park and Goble 2009, 559). While multidisciplinary working involves representatives from several disciplines 
contributing knowledge and methods from their fields to solve problems or carry out generalised interpretations (Austin, Park and Goble, 2009; Dogan and Pahre, 1990), interdisciplinary work is expected to go further and lead to an integrative level of understanding (Kokelmanns, 1979). Barry et al. (2008) highlight a number of alternative, contemporary approaches on interdisciplinarity and interdisciplinary working which challenge the "autonomy" of disciplines, particularly in the sciences (Nowotny et al. 2001). Haythornthwaite argues that central to the success of these approaches, is intensive collaboration leading to "new jointly determined definitions of work routines, technologies use and communication practice" but cautions that this involves the "active interrogation of invisible practices" and a surfacing of "specificities" as a prerequisite to collaborative work across pre-existing disciplinary boundaries (2006, 761-4). Strathern has argued that one outcome of this more emergent model of interdisciplinary working is the support of participants' reflection on their own existing disciplinary practices, leading to new conceptualisations of accountability and quality (2004).

These perspectives align interdisciplinary working within what Hakkarainen et al. $(2004,11)$ describe as the 'knowledge creation' metaphor for learning, which extends the 'acquisition' and 'participation' metaphors described by Sfard (1998) by involving the deliberate advancement of knowledge, tools and their associated social practices and discourses. Knowledge creation has to purposefully build on existing cultural and community knowledge, which needs to be exposed through the kinds of carefully structured enquiries described by Haythornthwaite and genres of 'conversation' described by Patel and Solomon (2009).

A further exploration of interdisciplinary working is provided by Hall (1999) who describes how 'cultures of inquiry' represent a useful framing of complex and 
contested domains in which participants have been differently 'disciplined' and have diverse (and potentially antithetical) standpoints and commitments. Hall contends that such a culture of enquiry can support the discursive construction of multiple narratives and that these are individually and collectively enriched and advanced through a process of formative critique. He describes (152-156) how the multiple historical interpretations of the British Civil Wars of the mid $17^{\text {th }}$ century (Marxist, Whig, economic, constitutional and other accounts) have benefited from such discourses. Key events or issues act as points of focus for conversation and critique of the accuracy and adequacy of accounts, and around contested concepts (a good example being the view, central to Marxist historians, that there was an 'English Revolution' - not a view shared by others). Hall then extends this into a model for social enquiry more generally, in which he maps out the 'generalising' and 'particularising' practices employed by researchers and the potential for these to intersect in ways that can contribute to collective understandings and the creation of new approaches.

So: the Ensemble project team's response both to the complexity of the project in general, and to its interdisciplinary character in particular, has been twofold. Firstly there has been a conceptualisation of the extended project as a culture of inquiry, characterised by 'conversations' and processes of collaborative interpretation. Secondly, these conversations (which have taken place through face to face discussions, team working, collaborative writing, and through the sharing of readings, resources and examples) have been directed to a series of points of focus (Wenger), specificities (Haythornthwaite) or generalising and particularising practices (Hall). Two of the areas that have promoted (and continue to promote) the most engaging discussions across the entire team are those of participation and ethical frameworks. 
Not only are these both complex and contested in their own right, the discourses around them have also raised 'creative tensions' - echoing Hall's description of formative critiques by one discourse of the other. The commitment of the project to an expansive and emergent idea of participation presented dilemmas and challenges when the project came to consider ethical frameworks and the specifics of the ethical review processes.

\section{The Participatory Practices of the Project}

The commitment of the project to be 'participatory' led to wide ranging discussions across and beyond the project, as it was discovered that this was an area of concern across other projects running concurrently within the Technology-Enhanced Learning programme. The project's understanding of participation has been a broad one. Like Elliot (2006) and Boser (2006) we have seen participatory approaches to research as involving engagement in all stages of the research project and in all key deliberations. As Boser states $(2006,14)$ : 'In contrast to conventional research, these approaches see those who would normally constitute "the researched" as actively involved in defining the questions, in data collection and analysis and in interpreting and taking action based on the research findings.'

These discussions drew on traditions of participatory action research, which are emergent in technology enhanced learning (Somekh 1997, 2001; Carmichael, 2003), and on ideas about participatory design, drawn from computer sciences and software development (Olsson 2004). A further current came from conceptions of participation informed by project members' experience of international development projects and their experience of participatory rural appraisal (Chambers 1994). 
What participatory action research offered was a framing of project activities, including, critically, the development of an ethical framework, not as being driven by well structured hypotheses but rather by dilemmas and challenges which are explored with the aim of gaining a deeper understanding and improvement in practice (Muñoz and Jeris 2005, 8). Hilsen $(2006,26)$ suggests that participatory action research cannot be reduced to an 'organisational recipe' ready to be implemented, but is rather an 'ever-present endeavour': new understandings and transformations in practice can and will happen throughout the process.

The action research that Elliott describes is not linked to any one research method or methodology; rather, methods are 'selected in the context of practice as the situation unfolds' $(2006,146)$. This opens the door for mixed methods approaches to be used in research so that a variety of disciplinary research practices can be encompassed. In the Ensemble project, data collection and interpretation processes informed by social and situated learning theories sit alongside discourse analysis and ethnography, and methods are blended and adapted to suit each as necessary. Elliott further suggests that methods should be chosen with the aim of helping practitioners to 'develop a reasoned capacity for action in the service of their educational values', bringing consideration of ethical issues into scope alongside other methodological deliberations $(2006,178)$. It is important to reflect that the methods and purposes of action research are themselves highly contested, and to argue that they are selfevidently 'fit for purpose' for interdisciplinary research activities would be misguided; Reason and Bradbury highlight the tendency for narrow interpretations of action research to emerge from particular disciplinary foundations: 'It upsets us when we see action research as narrowly drawn; when for example, we review an article that only sees action research as short-sighted consulting, seems to argue that one 
approach is the true form of action research, or traces action research back through just one discipline stream to one set of founding (usually masculine) authorities' $(2008,7)$. Participatory action research is not the 'answer' to a clearly defined set of questions then, but rather, in Hall's terms, needs to be understood as a set of particularising and generalising practices employed within multi-threaded project discourses.

A different perspective on 'participation' came from computer scientists within the project who recognised in the commitments of the project a need to employ ‘participatory design' approaches to software development and evaluation. Participatory design (PD) encompasses a wide range of approaches and 'degrees of user involvement' (Olsson 2004, 379). Muller et al. (1997) point out that some techniques are described as 'participatory' when participants are used 'simply as a source of information to be exploited, objectified or manipulated, thus reducing the whole process of participation to an illusion' (cited in Olsson 2004, 378). However, PD has also moved away from this simple empiricist view; not only is there increasing interest in the methods of social science, including action research (Gottesdiener 2002), there have also been moves to integrate perspectives that reconstruct technologies as social practice (Suchman et al., 1999; Suchman 2007). This extends beyond gathering 'user requirements' and design is understood to be a process of enquiry that extends beyond completion of a software project. It extends to exploration of the sense and use made of technologies once they become part of the social practices of different users, who are engaged as co-designers in a continuing process of development of both technology and practice.

Variability and adaptability are inherent by the very nature of participatory research and more expansive versions of participatory design. The need for 
participatory research to be adaptive to circumstances, to adopt different research methods and to engage with participants in different ways at different times makes it hard to predict potential issues in advance for the purposes of ethical review. BrydonMiller and Greenwood $(2006,119)$ describe action research projects as 'open-ended, collaborative, methodologically eclectic, and without specific methods, processes, or final goals determined in advance' and suggest that this makes it hard to review, evaluate and approve or disapprove of the ethical aspects of research plans at early stages of project development.

Our project commitments to engage participants across multiple settings and to allow them to contribute significantly to the framing of research questions and technological developments necessitated a flexible ethical framework which would, itself, develop through processes of formative critique from different disciplinary perspectives and from the participating teachers and students themselves. At the same time we had to address formal review processes not premised on such commitments and which were based on narrower conceptions and assumptions about the methods to be used, roles and relationships within the project and considerations of what counted as successful project outcomes.

We were concerned that our commitments were not inhibited by an overly protective culture of ethical review that might restrict emergent research practices on the grounds of the potential for unexpected ethical issues to arise during research. At the same time we were conscious of a very real possibility that what might emerge from processes of co-design of research and participatory design of technologies would go far beyond the initial plans scrutinised as part of an ethical review process and that ethical approval would have no bearing on the actual practices that developed. This could lead to concern on the part of funding bodies, host institutions, 
participants and research users as to the quality of the research and might even compromise the project. The challenge was to develop a set of practices, processes and materials which would serve both as a guide and a 'stabilised' performance of project commitments, while being flexible enough to allow methodological, pedagogical and technological innovation and participant input into project directions.

In the remainder of this paper, we will discuss two aspects of our response to this challenge: a specific 'enactment' of project commitments and approaches which was developed in order to address the expectations of initial ethical review boards; and a flexible and discursive approach to ethical practice which was employed in research settings. We characterise the latter as 'joint practical reasoning' which draws on Elliott's conception of participatory action research as the exercise of phronesis or 'practical wisdom'. It can also be seen as a particular instance of Hall's 'culture of inquiry', where the focus for the diverse and interdisciplinary project community is a developing ethical framework which is broader than any single, specific enactment.

\section{Ethical Framework and Ethical Review}

Discussions leading to the development of both a broad ethical framework and the specific documentation required for the review process began even before the project commenced its funded phase in October 2008. Previous experience of multi- and interdisciplinary working, together with the interest in promoting the continuing and interwoven discourses of a 'culture of enquiry' led to the organisation of a series of meetings at which the entire research team met over a period of several days and research ethics was a theme which was introduced and revisited regularly. In addition, related discussions about research settings, methods, research archiving, publication strategies, for example, frequently involved a revisiting of ethical issues 
and consideration of whether the emerging ethical framework would need to take account of the issues under discussion.

Within the culture of inquiry, an ethical narrative emerged as one of the 'discursive articulations of practice' described by Hall $(1999,171)$. Interestingly, despite the 'pandisciplinarity of enquiry' it proved useful for a member of the project team (one of the authors of this paper) to deliberately maintain this narrative by interrogating emerging plans based on prior experience of research and by offering 'plausible counterfactuals' (Hawthorn, cited in Hall 1999, 158). These were 'what if' questions and scenarios which posed a challenge to the project's current understandings of its ethical practice, or to a research method which might raise issues about what constituted 'informed consent' or which generated data that might make preservation of anonymity difficult. Many of these issues arose from the aspects of the project highlighted in the opening section of this paper: examples included the use of video and audio of classroom discussions - both whole class and small group working; the collection, in some settings, of online data generated as students interacted with online resources; and the potential for student learning opportunities to be affected (positively or negatively) by the use of new technologies. In some cases, difficulties emerged where project objectives were in tension with each other or with established ethical commitments: for example, the preservation of anonymity, attribution or informed consent. The project team had to reconcile the central role of data sharing (an intrinsic element of the semantic web's linked web of data) with commitments to data protection and privacy when these data were emerging from research settings. Similarly, the notion of informed consent had to be revisited where data was not simply being stored and retrieved for research purposes, but reanalysed, repurposed and combined in ways not obvious or articulated at the time that consent was 
originally given for their collection. The ethical framework acted in cases such as these, not to aid arbitration or decision-making. Instead, it provided a framing for questions to be raised about how our use and development of advanced technologies might represent a challenge to our ethical practice and those of research participants, and as a tool to encourage reflection as to how these tensions could be resolved. The formative nature of the discourse meant that questions were not couched in terms of 'would our ethical framework 'cover' this?' Rather, the questions took forms such as:

- 'What do our ethical commitments mean for the way in which we need to introduce and potentially adapt this research method?'

- 'Are the notions of privacy promised by this technological system sufficient that we can offer them with confidence to participants?'

- 'Is our ethical framework as currently constructed adequate to deal with a situation in which ...?'

- 'Are we sure that participants understand fully that this research method may have consequences in these areas ...?'

The preparation for the process of formal ethical review was clearly informed by these discussions, but at the same time represented the discursive generation of an additional narrative. In some ways this seemed like a translated and 'edited' version of a richer account informed by and informing, critiqued by and critiquing, other project narratives and discourses, but clearly an important interface between our emerging understandings and plans, and a more generalised notion of ethical practice.

The process of preparing for ethical review was complicated by the fact that we were conducting research in two universities with different review processes. In one (Institution A) a university-wide ethics board sat at regular intervals and 
considered proposals for research by and within the institution. In the other (Institution B), responsibility for ethical review was devolved to faculty-level boards, each with its own processes and procedures for reviewing research proposals. These differences were evident as we came to prepare the two sets of documentation for submission. In each case we were conscious that we were producing material enactments of a wide range of practices which were in some cases still highly contested within the project and which had yet to be presented, negotiated and elaborated in different research settings - like 'snapshots' of our current state of understanding, underpinned by a number of key concepts and commitments.

In Institution A, a set of documents common to all research involving 'human subjects' required an abstract of the research, a section on the 'research protocol ...to be used' and also included questions which, asked about the 'frequency of procedures'. For a research project only beginning to negotiate the nature of its engagement with participants, these questions proved difficult to answer. In Institution B, a three stage process was involved - the first involved our completion of a structured questionnaire designed to promote reflection on the part of the researchers, who were then asked to submit this with supporting documentation to a 'knowledgeable person of standing' identified by the faculty. The second stage of this process involved a meeting and discussion, with the option of progress to a third stage which would involve a full ethical review panel. The implications of certain responses for progress to further review processes was made clear, the accompanying notes reading:

"If you have [answered 'yes' to any of these questions] then you should assume that further discussion involving Stage 2 procedures is required because some aspect of your proposed research is likely to be 'ethically sensitive'. In practice, many issues can be resolved at this stage ... if you have [answered 'yes' to one or more of questions involving potential 
stress to participants or medical procedures] Stage 3 clearance will definitely be required".

In both cases, we submitted not only the formal documents provided by the institutions, but the current version of the broader project ethical framework, sample documents to be distributed to participants, and other project documentation. And in both cases, ethical approval for the project to proceed was given, Institution A's board asking for some clarifications about project oversight and resolution of disputes.

Following ethical approval from both institutions, members of the project team discussed the two processes and there was agreement that, while we were generally confident of the outcomes of the review processes, Institution B's structured process had been not only more transparent but that undergoing the preparation of the submission had contributed to our thinking about the ethical frameworks we might use. Not only was our collection of documentation and examples, a material enactment of our own (emergent) research practices and discourses; the structured but transparent questionnaire was itself an enactment of the faculty's own collective experience of the conduct of ethically sound research. By framing at least the early stages of the process as part of a discourse rather than as 'approval' or 'clearance', it contributed formatively to the ongoing culture of inquiry of the project, aligning well with our own practices and with the processes by which our broader ethical framework had been developed.

That said, there were elements of the more generalised review process of Institution A which were clearly designed to promote reflection on the part of researchers: including an open question asking "What do you consider are the major ethical issues in this proposal?" Even then, there was discussion within the project as to what issues should be offered and how these should be couched, and given that we saw the issue of 'informed consent' as one of our major areas for discussion with 
participants it was felt appropriate to mention this. But as one of the preceding questions asked "How and when will [participants'] consent be obtained?" our response - that the obtaining of consent would be regarded, not as a once-and-for-all prior event, but as a process, subject to renegotiation over time - was supported with a copy of our full ethical framework and copies of the template for consent forms which had been developed for use in project research settings.

Therefore the 'single discipline' review process of Institution B did encourage group discourse relating to appropriate educational research ethical issues, but this did not encompass the full range of concerns that an interdisciplinary technologyenhanced project may face. The broader ethical review of Institution A attempted to cover a wide range of disciplines or be discipline-independent, which invited interdisciplinary thinking and discussion - but it wasn't framed as part of an ethical discourse. The challenge for ethical review boards may therefore be to integrate the approaches of these two institutions with questions that initiate discussion and reflection on ethical issues and practices, whilst remaining open and wide ranging enough to allow for interdisciplinary thinking.

\section{Research Ethics as 'Practical Wisdom'}

These different processes of ethical review, combined with a recognition of the importance of personal experiences (both of engaging with ethical issues and of research practices which might raise them) that are enriched through working as an interdisciplinary group, led us to explore in more detail the relationship between two of the elements in our culture of inquiry: namely the ethical narrative and that of participation which has been outlined above. 
In such an interdisciplinary project, and particularly given that the extended project team includes participants from widely diverse disciplines, it is unlikely that the research aims of all project participants will fit simply into either of Elliott's (1978) characterisations of 'educational research' (which aligns with participatory action research and involves the practical intention to realise educational values) and 'research on education' (which aspires to produce "objective knowledge" about educational practice). However, it is clearly important that researchers reflect on the aims and underpinnings of their research and these are shared with the project as a whole, opening up opportunities for discussion about methodologies, practices and, of course, ethical issues. Thus project discussions in which ethical issues are regularly revisited are key to conducting ethical research and realising Elliott's 'ethically committed action'. Echoing but also extending our understanding of the 'culture of inquiry', Elliott argues this is premised on 'disciplined conversation in which reasons for action are scrutinized, critiqued and modified' and connects this approach with the Aristotelian concept of phronesis as a distinctive form of ethically informed practical reasoning.

To possess practical wisdom in Aristotle's view is to be good at thinking about what one 'should' do, and involves a combination of understanding and experience that allows one to make judgements in new situations (Hughes 2001, 84). Phronesis is one of three principle intellectual virtues proposed by Aristotle (2000), the other two being episteme and techne. Episteme is classed as scientific knowledge of eternal and universal truths (Aristotle 2000, 105). In contrast, techne is translated as art, craft or skill; the ability to 'bring into being things that are contingent and variable' (Wiliam 2008, 434). Phronesis transcends both episteme and techne as it concerns the problem of acting rationally in situations that are contingent and variable (Wiliam 2008, 434), 
and therefore allows responses to situations that are complex and interdisciplinary working is developing.

Hughes $(2001,84)$ interprets Aristotle's concept of phronesis as including the following requirements and actions. Phronesis involves assessing each potential action in the light of the particular situation, therefore:

- Ethical frameworks cannot prescribe actions, only principles for consideration. They lay the foundations for ethical practice but each new situation or research relationship requires re-assessment of those codes in the light of the circumstances.

- The implications for ethical review processes are that it is not possible to prescribe practical ethical solutions to all imagined future issues. Attempts to pre-empt all problems and their solutions could lead to synthetic and potentially irrelevant statements being made in review documentation.

In the context of the Ensemble project, this involved the 'tailoring' of the ethical framework to take account of particular settings and situations. In one research setting, student use of an online learning environment was automatically 'tracked', generating detailed data about their behaviour and interactions. While this had not been explicitly mentioned in the original ethical framework, it was possible to extend this in a way that aligned with the project's general commitments to informed consent, and participating students were made aware of this additional aspect of data collection relating to them.

Phronesis also requires an understanding of what the underlying moral principles are and also the experience to use that understanding correctly, so: 
- Ethical frameworks are not sufficient in themselves to prescribe actions. In order to make ethical decisions, you need to have enough experience of the potential outcomes to make a judgement about the best course of action.

- Having an interdisciplinary group of researchers with varying levels of experience can be used as a resource for making ethical judgements.

In one of the Ensemble project research settings, technological innovations were gradually introduced within small elements of an undergraduate course, rather than introducing a range of new and untested technologies more broadly. This was underpinned by a commitment on the part of both researchers and teachers to a 'duty of care' towards students and a desire not to put them at risk in a 'high stakes' learning and assessment environment.

Phronesis, according to Hughes, also requires a combination of understanding and experience that is continually being enhanced through confrontations with different situations. In the context of interdisciplinary research, this means:

- Researchers and groups can benefit from reflecting on and sharing their experiences of ethical issues and the outcomes of the actions put into place to solve them.

- The interdisciplinary working and the culture of inquiry, which the project seeks to establish, should see the diverse and developing experience of participants as a resource upon which to draw in addressing ethical issues, rather than as a barrier to understanding.

In the course of project activities, we initially attempted to gather data about student case-based learning in one research setting by providing them with a range of ethnographic 'probes' for self-documentation (audio recorders, video and still 
cameras, diaries). Even with the full cooperation of students, the data collected offered limited possibilities for analysis. Learning from this, in a second setting, we approached students with the proposal that they be accompanied or 'shadowed' by a researcher who would collect data on their activities. We had previously assumed that students would be less willing to agree to this, but in fact they indicated that the presence of the researcher would, in fact, be less intrusive than the demands of selfdocumentation. This change in approach actually resolved one area of concern, namely whether the participating students would themselves carry out data collection with due regard for the project's ethical commitments, but at the same time, necessitated a further renegotiation of the ethical framework and informed consent arrangements in the light of the new research relationships in that particular setting.

A phronetic approach to educational research involves more than a broad commitment to collegiality or democracy; and an open and democratic approach to practicing research does not necessarily imply consensus in the moral aims of conducting research. As Boser points out 'democratic intentions do not obviate the need for thoughtful examination of the ethical implications' of our research (2001, 14). So ethical issues need to remain open questions, revisited, as research practices, technologies and relationships develop, and thoughtfully reassessed in the light of new understandings. In Hall's terms, the other narratives (disciplinary, pedagogical and technological) should always remain capable of challenging ethical practices, just as they should be subject to scrutiny, critique and modification in the light of emerging ethical concerns. 


\section{Practical Wisdom, Interdisciplinary Working and Critique in Research Settings}

We have alluded to an extended set of ethical 'materials' which have emerged from discussions, informed by prior experience and disciplinary perspectives, and which accompanied the more constrained and structured ethical review documentation. We conclude this paper with a vignette of project engagement in one research setting, describing some aspects of the ethical issues involved and highlighting the role of the context-specific, formative discourses in which researchers and participants have taken part.

The setting is a postgraduate course in one of the participating universities in which the complexity of the subject matter, and the interdisciplinary nature of the field (which draws on technology, business, management, law and politics), makes learning with cases the dominant pedagogical approach. Students with diverse backgrounds engage with a series of presentations, films and other resources before working in groups to solve problems presented by the teacher. The teaching and learning environment has, until now, used few technologies, so introduction of the semantic web technologies into this setting is necessarily proceeding slowly - the teacher and students prize the team working and face-to-face negotiation skills that will prepare them for future employment.

When project researchers first visited the classroom to observe teacher and students at work, it followed a series of discussions about the nature and scope of the projects' involvement, expectations and issues of confidentiality and privacy. The student group were then given an opportunity to discuss these issues, and were presented with a leaflet about the project, a tailored copy of the project's ethical framework, and a customised consent form which they were given an opportunity to discuss with the teacher and researchers. The project's documentation includes a 
series of consent form 'templates' which set out in a clear format, for example, what data are to be collected, from whom and for what purpose. These templates are customized to reflect both the nature of the setting and the outcomes of these discussions with teachers and students (including the detailed cases outlined above). These were the original documents submitted to the ethical review boards - but once adapted, each setting, and potentially each research encounter (interview, discussion or classroom observation) could have slightly different, context-sensitive material reflecting different (and changing) notions of informed consent.

Ethical issues, and particularly that of informed consent, are never taken for granted, and are the subject of continuing discussion. What has occurred, however, is more than a 'customized' set of ethical practices enacted by the research team; adoption of a phronetic problem-solving approach means that the different disciplinary perspectives and varied experience represented, offer not only creative solutions to dilemmas, but are also a source of critique of the ethical framework itself, which continues to evolve as a result. And these critiques come not only from within the project team; in the research setting we describe here, the teacher challenged the way in which we were conceptualising confidentiality. We had considered confidentiality primarily in terms of protection of individuals' privacy but the teacher argued that given the business orientation of the course concerned and the fact that classroom contributions might represent privileged information and particular learning outcomes might mean competitive advantage, we needed to adapt and extend our understanding in this area. This led us to revisit not only data collection protocols but also our thinking about long-term storage of data and the incorporation of primary classroom data into publications. 
A more complex relationship between the ethical, pedagogical and technological strands of the project's work also emerged in the course of discussions with students about the collection of video data; this is a necessarily brief summary, but it captures both the participatory nature of the project, the potential for unexpected developments and the ways in which the different strands and narratives of the project intertwine:

- Teachers and students agreed that researchers could collect video data both of lectures and of group work as students worked on cases; students who were reticent were reassured that the video was being collected for specific research purposes - fine grained discourse analysis.

- By the end of the course, student curiosity as to what had been observed had evolved into a realisation that the videos might be of value in supporting their own learning, either as records of lectures, as reminders of their own contributions, or as opportunities for 'vicarious learning' by observing others.

- The teacher was also interested and was increasingly reflecting on his own practice and on exploring how students worked in small groups, solving problems and introducing knowledge from different sources into discussions.

- The ethical framework in this specific example was renegotiated so that teacher and students were able to view the videos in a specially established secure online environment, with the agreement of all concerned. This negotiation was not an easy one and the implications were particularly carefully explored; the prior experience of project members of supporting secure online spaces and establishing linked ethical frameworks (Carmichael and Youdell, 2007; Wilson et al. 2007) was particularly important and strengthened participants' confidence in the solution offered. 
This vignette shows how a commitment to participation and the phronetic principle of drawing upon both understanding and experience in order to decide how to act, allowed a creative solution to a complex and emergent problem. At the same time, it demonstrates how a single narrative (technological, pedagogical, ethical) cannot adequately express or inform a situation in which: an unforeseen affordance of a technologically enhanced research process contributed to changed pedagogical and andragogical practices; these necessitated a new technological aspect to the learning environment; and a renegotiation of associated ethical practices. These experiences, pedagogical, technological and ethical, are now available to the project researchers as they engage with other, different settings and can inform the next phase of engagement and ethical framing of research.

The online environment in which the teacher and students can engage with research data represents an enactment of ethical commitments as much (and very possibly more than?) the preparation of documents for formal ethical review. The emergence of new practices and the construction of new knowledge in complex, interdisciplinary and participatory research environments can be enabled and supported if ethical practice in research is understood as a series of open questions which are continuously scrutinized and critiqued from multiple perspectives. Ethical review processes are most valuable if they are understood not as 'approval' or 'clearance' but as contributing to, or even initiating, formative and dialogic practice.

\section{References}

Aristotle (2000) The Nicomachean Ethics (Translated by R. Crisp), Cambridge, UK: Cambridge University Press

Austin, W., Park, C., Goble, E. (2008) From Interdisciplinary to Transdisciplinary Research: A Case Study Qualitative Health Research 18 (4) 557-564

Barry, A., Born, G. and Weszkalnys, G. (2008) 'Logics of Interdisciplinarity' Economy and Society 37(1) 20-49 
Berners-Lee, T., Hendler, J. and Lassila, O. (2001) 'The Semantic Web' Scientific American May 2001 34-43

Boser, S. (2006) 'Ethics and power in community-campus partnerships for research' Action Research 4 (1) 9-21

Brydon-Miller, M., and Greenwood, D. (2006) 'A re-examination of the relationship between action research and human subjects review processes' Action Research 4 (1): $117-128$

Carmichael, P. (2003) Teachers as researchers and teachers as software developers: how use-case analysis helps build better educational software The Curriculum Journal 14(1) 105-122

Carmichael, P. and Youdell, D. (2007) Using Virtual Collaboration Environments for Education Research: Some Ethical Considerations Research Intelligence 100 26-29

Chambers, R. (1994) 'Participatory Rural Appraisal (PRA): Analysis of Experience' World Development 22(9) 1253-1268

Dogan, M., and Pahre, R. (1990) Creative marginality: Innovation at the intersection of social sciences. Boulder, CO: Westview.

Elliott, J. (1978) Classroom Research: Science or Commonsense, in: R. McAleese and D. Hamilton (eds) Understanding Classroom Life (Windsor, NFER Publishing Company)

Elliott, J. (2006) Educational Research as a Form of Democratic Rationality Journal of Philosophy of Education 40(2) 169-185

Feuer, M. J., Towne, L. and Shavelson, R. J. (2002) 'Scientific culture and educational research' Educational Researcher 31(8) 4-14

Gottesdiener, E. (2002) Requirements through Collaboration (Addison-Wesley, Boston MA)

Hakkarainen, K., Palonen, T., Paavola, S., and Lehtinen, E. (2004). Communities of networked expertise: Professional and educational perspectives (Elsevier, Amsterdam)

Hall, J. (1999) Cultures of inquiry: From epistemology to discourse in sociocultural research (Cambridge University Press, Cambridge)

Hawthorn, G. (1991) Plausible Worlds: possibility and understanding in history and social science (Cambridge University Press, New York)

Haythornthwaite, C. (2006) Articulating divides in distributed knowledge practice. Information, Communication \& Society 9(6) 761-780

Hilsen, A.I. (2006) And they shall be known by their deeds: Ethics and politics in action research. Action Research 4 (1) 23- 34

Hughes, G.J. (2001) Aristotle on Ethics (Routledge, London)

Kokelmanns, J.J. (1979) 'Why interdisciplinarity?’ in J.J. Kokelmanns (Ed.)

Interdisciplinarity and higher education (London, Pennsylvania State University Press) 
Muller, M.J., Haslwanter, J.H., Dayton, T., (1997) Participatory practices in the software lifecycle. In: Helander, M., Landauer, T.K., Padhu, P. (Eds.), Handbook of Human - Computer Interaction (Elsevier, Amsterdam)

Muñoz, K., Jeris, L. (2005) Learning to be interdisciplinary: An action research approach to boundary spanning Health Education Journal 64 (1) 5-12

Nowotny, H., Scott, P. and Gibbons, M. (2001) Rethinking Science: knowledge and the public on an age of uncertainty (Cambridge, Polity Press)

Olsson, E. (2004) What active users and designers contribute in the design process Interacting with Computers 16 377-401

Patel, U., Solomon, N. (2009) Conversations across Research Projects on Simulation: Uncovering the New Worlds of Work, Learning and Technology CRLL Conference, Lifelong Learning Revisited: What Next? University of Stirling, 24-26 June 2009.

Pollard, A. (2007) 'The UK's Teaching and Learning Research Programme: findings and significance' British Educational Research Journal 33(5) 639-804

Reason, P. and Bradbury, H. (2008) 'Introduction' in: The SAGE Handbook of Action Research (1-11) (SAGE Publications Ltd, London)

Rimpilainen, S. and Edwards, R. (2009) 'The ANTics of Educational Research: Researching Case-based Learning through Objects and Texts' Paper presented at CRESC Conference, Manchester, September 2009

Sfard, A. (1998). On two metaphors for learning and on the dangers of choosing just one Educational Researcher, 27 (2) 4-13

Shadbolt, N., Berners-Lee, T. and Hall, W. (2006) The Semantic Web Revisited IEEE Intelligent Systems 21(3) 96-101

Somekh, B. (1997) 'Classroom investigations: exploring and evaluating how IT can support learning' in: Somekh, B. and Davis, N. (Eds.) Using IT effectively in teaching and learning: studies in pre-service and in-service teacher education. (Routledge, London)

Somekh, B. (2001) 'Methodological issues is identifying and describing the way knowledge is constructed with and without information and communications technology Journal of Information Technology for Teacher Education 10 (1-2) 157178

Suchman, L., Blomberg, J. Orr, J. E. and Trigg, R. (1999) Reconstructing Technologies as Social Practice American Behavioral Scientist 43 (3) 392-408

Suchman, L. (2007) Human-Machine Reconfigurations: plans and situated actions (Cambridge University Press, Cambridge)

Strathern, M. (2004) Commons and Borderlands (Sean Kingston Publishing, Wantage)

Tscholl, M., Tracy, F. and Carmichael, P. (2009) 'Case methods, pedagogical innovation and semantic technologies' Paper presented at EC-TEL Conference, Nice, October 2009

Wenger, E. (1998) Communities of Practice: Learning, Meaning and Identity (Cambridge, Cambridge University Press) 
Wiliam, D. (2008) Comments on Bulterman-Bos: What Should Education Research Do, and How Should It Do It? Educational Researcher 37 (7) 432-438

Wilson, A., Rimpilainen, S., Skinner, D., Cassidy, C., Christie, D., Coutts, N. and Sinclair, C. (2007) Using a Virtual Research Environment to support new models of collaborative and participative research in Scottish education Technology, Pedagogy and Education 16(3) 289-304 